\title{
Secure Transmission in Cell-Free Massive MIMO with RF Impairments and Low-Resolution ADCs/DACs
}

\author{
Xianyu Zhang, Tao Liang, Kang An, Gan Zheng, Fellow, IEEE, and Symeon Chatzinotas, Senior Member, IEEE
}

\begin{abstract}
This paper considers the secure transmission in a cell-free massive MIMO system with imperfect radio frequency (RF) chains and low-resolution analog-to-digital converters (ADCs) and digital-to-analog converters (DACs) at both access points (APs) and legitimate users, where an active eavesdropper attempts to wiretap the confidential data. The Gaussian RF impairment model (GRFIM) and additive quantization noise model (AQNM) are used to evaluate the impacts of the RF impairments and low resolution ADCs/DACs, respectively. The analytical results of the linear minimum mean square error (MMSE) channel estimation show that there is nonzero floor on the estimation error with respect to the RF impairments, ADC/DAC precision and the pilot power of the eavesdropper which is different from the conventional case with perfect transceiver. Then, a tractable closed-form expression for the ergodic secrecy rate is obtained with respect to key system parameters, such as the antenna number per AP, the AP number, user number, quality factors of the ADC/DAC and the RF chain, pilot signal power of the eavesdropper, etc. Moreover, a compensation algorithm between the imperfect RF components and the inexpensive coarse ADCs/DACs is also presented. Finally, numerical results are provided to illustrate the efficiency of the achieved expressions and the devised algorithm, and show the effects of RF impairments and low resolution ADC/DAC on the secrecy performance.
\end{abstract}

Index Terms-Cell-free massive MIMO, physical layer security, RF impairments, low-resolution ADCs, low-resolution DACs, active eavesdropping.

\section{INTRODUCTION}

$\mathbf{M}$ ASSIVE multiple-input multiple-output (MIMO) is an emerging paradigm for the fifth generation $(5 \mathrm{G})$ and beyond fifth generation (B5G) wireless networks, which can provide significant spectral and energy efficiency by deploying a large number of antennas at the base station or user equipment [1], [2]. However, deployment of large-scale antennas on a single base station, i.e., co-located massive MIMO, is a major challenge in practical deployment, whose huge antenna array

This work is supported by the National Natural Science Foundation of China under Grant 61901502 and U19B214, in part by the UK Engineering and Physical Sciences Research Council (EPSRC) under the grant EP/N007840/1. (Corresponding author: Kang An)

Xianyu Zhang, Tao Liang and Kang An are with the Sixth-third/63rd Research Institute, National University of Defense Technology, Nanjing, 210007 China. (e-mail: zhangxy_sat@126.com, liangtao63@sina.com, ankang89@nudt.edu.cn.)

G. Zheng is with the Wolfson School of Mechanical, Electrical, and Manufacturing Engineering, Loughborough University, Loughborough, U.K. (e-mail: g.zheng@lboro.ac.uk).

S. Chatzinotas is with the Interdisciplinary Centre for Security, Reliability and Trust, University of Luxembourg, Luxembourg (e-mail: symeon.chatzinotas@uni.lu.). will also lead to high channel correlation [3]. Distributed massive MIMO is a promising alternative which installs several distributed antenna sets onto different geographical locations [4]. As a scalable implementation of the distributed system, cell-free massive MIMO can provide significant advantages in terms of throughput and service coverage through a larger number of geographically distributed access points (APs) [5], [6]. Recently, various contributions in existing literatures, including performance analysis, full-duplex system, relaying communication, power allocation etc, have been presented on cell-free massive MIMO systems [7], [8], [9], [10].

Due to the open nature of the wireless channels, security is a critical issue in wireless communication systems [11]. As a complement of conventional cryptography techniques, physical layer security can be exploited to enhance secure wireless transmission, which has attracted significant research interest in recent years [12]. Because of the abundance of antennas, massive MIMO has the potential in improving physical layer security [13]. Until now, several works have been presented on physical layer security in co-located massive MIMO system, such as multi-cell system, relay massive MIMO system, etc [14], [15], [16], [17], [18]. In addition, secure transmit and security-constrained power allocation in multi-user distributed massive MIMO system have been investigated in [19] and [20]. For cell-free massive MIMO systems, the authors in [21] studied the secure transmission and power control under active pilot spoofing attacks. Besides, the secure communication in multigroup multicasting cell-free massive MIMO systems against active spoofing attack has been investigated in [22]. Secure transmission for simultaneous wireless information and power transfer (SWIPT) cell-free massive MIMO with active eavesdropper has been studied in [23].

Most prior works adopted a common assumption that all APs and users are equipped with perfect radio frequency (RF) chains, analog-to-digital converters (ADCs) and digital-toanalog converters (DACs). However, with the increase of APs in cell-free massive MIMO systems, applying high-quality hardware and high resolution ADCs/DACs bring significant financial costs and energy consumption. From the security perspective, the impacts of hardware impairments on secure co-located massive MIMO systems have been studied in detail in [24] and [25]. In addition, the effect of RF hardware impairments on the physical layer security for cell-free Massive MIMO network has been investigated in [26]. The authors in [27] studied the secrecy performance of the massive MIMO system with low resolution $\mathrm{ADCs} / \mathrm{DACs}$ in the presence of 
pilot spoofing attack. The utilization of imperfect transceivers and economical coarse ADCs/DACs is a feasible solution to tackle the bottleneck of cost and energy restriction. For colocated massive MIMO system, the authors in [28] analyzed the impacts of both RF impairments and ADCs distortions on the channel estimation, capacity, energy efficiency as well as effective compensation algorithms. However, due to general impairment model, there were still residual impairments which should be further incorporated. Utilizing an additive quantization noise model and an extended error vector magnitude model, the uplink achievable rate of massive MIMO system with RF impairments and low resolution ADC has been derived in [29]. Besides, the authors in [30] investigated the uplink achievable rate by considering both the nonideal RF chains and low-resolution ADCs in cell-free massive MIMO system. However, to the best of our knowledge, the secure communication in cell-free massive MIMO system with RF impairments and ADCs/DACs imperfections is still an open issue.

Motivated by the aforementioned observations, this paper considers the secure communication in cell-free massive MIMO system with RF impairments and ADCs/DACs imperfections. The Gaussian RF impairment model (GRFIM) and additive quantization noise model (AQNM) are advocated as suitable models for the RF impairments and ADCs/DACs imperfections, based on which the linear minimum mean square error (MMSE) algorithm is presented for channel estimation [31], [32]. With the adopted RF impairments and ADC imperfections models along with the imperfect channel state information (CSI) assumptions, the closed-form expression of the ergodic secrecy rate has been derived in terms of key design parameters, i.e. pilot and data power, the quality of the RF chains, the precision of the ADCs/DACs, the number of APs and antennas. Besides, the compensation between RF impairments and ADCs/DACs imperfections has been considered and illustrated by simulations. Unlike existing works on secure communication in massive MIMO system which simply considers specific architecture, this paper studies the secure transmission in cell-free massive MIMO sytem with a universal paradigm taking into account the nonideal RF chains, ADCs/DACs distortions and CSI imperfection. The key contributions of this paper are summarized as follows:

- Using the GRFIM and AQNM to model the RF impairments and the ADCs/DACs imperfections, the linear MMSE algorithm is utilized for uplink channel estimation at each AP locally. Based on which, the closedform statistical properties of the channel estimation and estimation error are provided. Subsequently, each AP can operate the downlink data transmission with the estimated channels.

- The closed-form expressions for the achievable rate of the target user and the Eve are presented, respectively. Then, the closed-form result of the ergodic secrecy rate is derived with respect to key system parameters, such as pilot power, the number of legitimate users and the total antennas, the RF quality factor, the quantization bit of the $\mathrm{ADCs} / \mathrm{DACs}$, etc. In general, the closed-form expression can provide generalized and effective insights to evaluate the system parameters on the secrecy performance of the considered system.

- To effectively investigate the impacts of the hardware impairments, the compensations between RF impairments and the low resolution ADCs/DACs has been studied in details. That is, different hardware assembling schemes by adjusting the quality factors of the RF chains and ADCs/DACs can achieve the same system performance. Furthermore, an iterative algorithm on performance compensations has been provided and illustrated by simulations.

The outline of the paper is organized as follows. Section II describes the considered secure cell-free massive MIMO system model. Then, the uplink linear MMSE channel estimation is presented in Section III. In Section IV, the downlink data transmission, secrecy performance analysis, and the performance compensations between the RF impairments and coarse ADCs/DACs are discussed in detail. Next, the simulation results are provided in Section V. Finally, Section VI concludes this paper.

Notation: Throughout the paper, boldface lowercase letters and uppercase letters denote vectors and matrices, e.g. g, G. The superscript $(\cdot)^{T},(\cdot)^{*}$ and $(\cdot)^{H}$ express the transpose, conjugate and Hermitian transpose, respectively. $\|\mathbf{g}\|(|g|)$ represents the the Euclidean norm (absolute value) of a vector g (a scalar $g$ ). $\mathbb{E}\{\cdot\}$ denote operations of expectation. diag (g) is a diagonal matrix consisting of diagonal entries of vector g. $[\mathbf{G}]_{i}\left([\mathbf{g}]_{i}\right)$ represents the $i$ th column vector of the matrix $\mathbf{G}$ (the $i$ th element of the vector $\mathbf{g}$ ). A circularly symmetric complex Gaussian random vector $\mathbf{x}$ with mean $\mathbf{m}$ and covariance matrix $\mathbf{A}$ can be represented by $\mathbf{x} \sim \mathcal{C N}(\mathbf{m}, \mathbf{A}) . \mathbf{I}_{M}$ is $M$-dimensional identity matrix. $\mathbb{C}^{M \times N}$ stands for $M \times N$ dimensional complex space. Besides, i.i.d indicates the abbreviation of independent and identically distributed. RV is the abbreviation of random variable. Finally, $[x]^{+}=\max \{0, x\}$.

\section{SYSTEM MODEL}

As illustrated in Fig. 1, this paper considers a cell-free massive MIMO communication system with $M$ APs (each equipped with $N$ antennas) and $K$ single antenna users in the presence of an active single antenna eavesdropper (Eve) which are all randomly positioned in a certain region. All APs simultaneously serve the $K$ users with the same time-frequency resource and are connected to a central processing unit (CPU) via error-free fronthaul links. By operating in time-division duplex (TDD) mode, the APs can achieve the CSI by uplink training by using the channel reciprocity between the uplink and downlink channels. Similar to existing literatures [5], [7], [21], [22], [26], [31], the uncorrelated flat Rayleigh fading channels are advocated in this paper. Specifically, the channel vector between the $m$ th $\mathrm{AP}$ and the $k_{1}$ th $\left(k_{1}=1,2, \cdots, K\right)$ user can be denoted by

$$
\mathbf{g}_{m k_{1}}=\sqrt{\beta_{m k_{1}}} \mathbf{h}_{m k_{1}},
$$

where $\beta_{m k_{1}}$ represents the large-scale fading including path loss and shadowing effects, $\mathbf{h}_{m k_{1}} \in \mathbb{C}^{N \times 1}$ models the smallscale fading vector with elements being i.i.d. $\mathcal{C N}(0,1)$ RVs. 


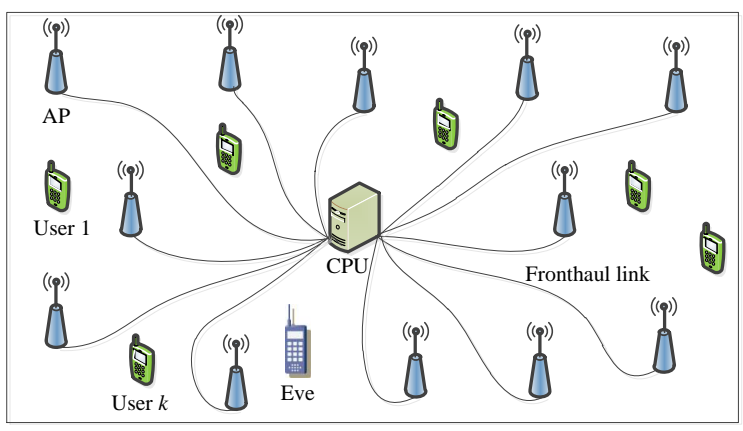

Fig. 1. Illustration of the secure cell-free massive MIMO system.

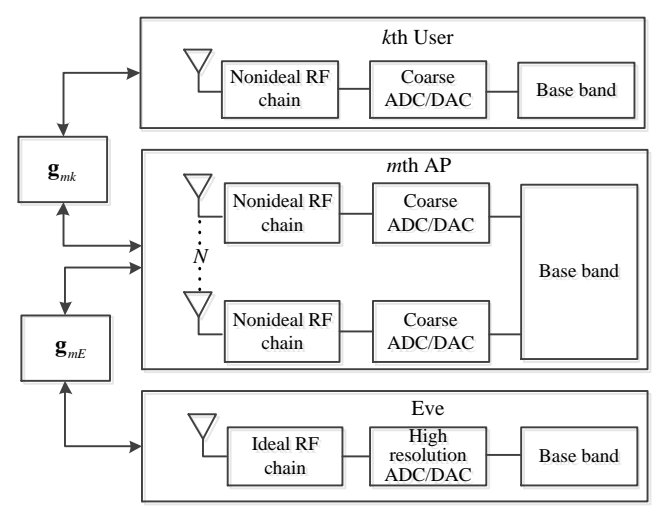

Fig. 2. Architecture of the AP, legitimate user and the Eve.

Besides, the channel between the $m$ th $\mathrm{AP}$ and the active eavesdropper (Eve) is given by

$$
\mathbf{g}_{m E}=\sqrt{\beta_{m E}} \mathbf{h}_{m E},
$$

where $\beta_{m E}$ is the large-scale fading coefficient, $\mathbf{h}_{m E} \sim$ $\mathcal{C N}\left(\mathbf{0}, \mathbf{I}_{N}\right)$ denotes the corresponding small-scale fading vector.

To further reduce the implementation costs and energy dissipation in cell-free massive MIMO system, this paper considers the economical coarse RF chains and low resolution ADCs/DACs at both the APs and the legitimate users. Furthermore, the eavesdropper can enhance its wiretapping capability by equipping with high quality transceivers and high resolution ADCs/DACs. Hence, this paper makes a worst-case assumption that the Eve is equipped with perfect hardware and ideal ADCs/DACs. The architecture of the APs, legitimate user and the Eve is depicted in Fig. 2.

The secure downlink transmission is considered in this paper. Hence, one coherence interval includes two phases: uplink channel estimation and downlink data transmission.

\section{UPLINK CHANNEL ESTIMATION}

This phase includes the channel estimation through uplink pilots. The large-scale attenuations are assumed constant within each coherence interval and known a priori. Nevertheless, the small-scale fading gains are unknown and needed to be estimated. All $K$ legitimate users simultaneously send pilot sequence to the $M$ APs. Let $\tau$ be the uplink pilot sequence duration (in symbols). The pilot signal used by the $k$ th user can be denoted by $\sqrt{\tau} \varphi_{k} \in \mathbb{C}^{\tau \times 1}$ with $\left\|\varphi_{k}\right\|^{2}=1$. Let $P_{k}$ be the set of the user indexes which utilize the same pilot signal as the $k$ th user. As a complication in massive MIMO system, the pilot contamination is considered in this paper, that is, different users may utilize the same pilot sequence, i.e. $\tau<K$. Since the pilot sequences are public and repeatedly used, a smart eavesdropper can transmit the same pilot as the target user. Without loss of generality, an assumption can be made that the Eve attempts to wiretap the confidential message to the $k$ th user, i.e. $\varphi_{E}=\varphi_{k}$. With an ideal hardware transceiver, the received pilot signal at the $m$ th AP can be represented by

$$
\mathbf{Y}_{p m}=\sum_{k_{1}=1}^{K} \sqrt{\tau p_{p}} \mathbf{g}_{m k_{1}} \boldsymbol{\varphi}_{k_{1}}^{H}+\sqrt{\tau p_{E}} \mathbf{g}_{m E} \boldsymbol{\varphi}_{k}^{H}+\mathbf{W}_{p m}
$$

where $p_{p}$ and $p_{E}$ denote the average transmit pilot power of the legitimate user and the Eve, and $\mathbf{W}_{p m} \in \mathbb{C}^{N \times \tau}$ is the additive white Gaussian noise (AWGN) with elements are i.i.d $\mathcal{C N}\left(0, \delta^{2}\right)$ RVs.

Using the well-established Gaussian RF impairment model (GRFIM) to evaluate the impacts of the coarse RF chains at the users and the APs [32], the received signal can be rewritten as

$$
\begin{aligned}
\mathbf{Y}_{p m}= & \sqrt{k_{A}} \sum_{k_{1}=1}^{K} \mathbf{g}_{m k_{1}}\left(\sqrt{\tau p_{p} k_{U}} \boldsymbol{\varphi}_{k_{1}}^{H}+\boldsymbol{\eta}_{k_{1} t}^{T}\right) \\
& +\sqrt{\tau p_{E} k_{A}} \mathbf{g}_{m E} \boldsymbol{\varphi}_{k}^{H}+\mathbf{D}_{m r}+\mathbf{W}_{p m},
\end{aligned}
$$

where $k_{U} \in[0,1]$ and $k_{A} \in[0,1]$ represent the $\mathrm{R}$ $\mathrm{F}$ hardware quality coefficients of the legitimate user$\mathbf{s}$ and the APs, respectively. $\boldsymbol{\eta}_{k_{1} t}$ and $\mathbf{D}_{m r}$ model the distortion noise at the transceiver of the $k_{1}$ th user and the $m$ th AP which are distributed as $\boldsymbol{\eta}_{k_{1} t} \sim$ $\mathcal{C N}\left(0, p_{p}\left(1-k_{U}\right) \mathbf{I}_{\tau}\right)$ and $\left[\mathbf{D}_{m r}\right]_{i} \sim \mathcal{C N}\left(0,\left(1-k_{A}\right) \Theta\right)$, $1 \leq i \leq \tau$, where the diagonal matrix $\Theta$ can be represented as $\Theta=p_{E} \operatorname{diag}\left(\left|\left[\mathbf{g}_{m E}\right]_{1}\right|^{2}, \cdots,\left|\left[\mathbf{g}_{m E}\right]_{N}\right|^{2}\right)+$ $p_{p} \sum_{k_{1}=1}^{K} \operatorname{diag}\left(\left|\left[\mathbf{g}_{m k_{1}}\right]_{1}\right|^{2}, \cdots,\left|\left[\mathbf{g}_{m k_{1}}\right]_{N}\right|^{2}\right)$.

For analytical tractability, the popular additive quantization noise model (AQNM) is utilized to quantize the output of the poor-resolution DAC[31]. Furthermore, the received signal through the low-resolution DAC at the $m$ th AP is given by

$$
\tilde{\mathbf{Y}}_{p m}=\alpha_{A} \mathbf{Y}_{p m}+\tilde{\mathbf{W}}_{p m}
$$

where $\alpha_{A}$ is the quantization factor at the APs which is determined by the quantization bits number of the DAC, $b_{A}$. Besides, $\tilde{\mathbf{W}}_{p m}$ is the additive Gaussian quantization noise at the $m$ th AP whose covariance matrix is approximated as [30], [33], [34]

$$
\mathbf{R}_{\tilde{\mathbf{W}}_{p m}} \approx \alpha_{A}\left(1-\alpha_{A}\right) \operatorname{diag}\left(\mathbb{E}\left\{\mathbf{Y}_{p m} \mathbf{Y}_{p m}^{H}\right\}\right) .
$$

Furthermore, all APs are assumed to be equipped with the same ADCs. The values of $\alpha_{A}$ are discrete and related to quantization bits $b_{A}$. For $b_{A} \leq 5$, the accurate values of $\alpha_{A}$ can be checked in Table I in [35]. While for $b_{A}>5, \alpha_{A}$ can be approximated as

$$
\alpha_{A}=1-\frac{\pi \sqrt{3}}{2} 2^{-2 b_{A}} .
$$


Then, projecting $\tilde{\mathbf{Y}}_{p m}$ onto $\varphi_{k^{\prime}}$, the post-processing signal can be given by

$\tilde{\mathbf{y}}_{p m, k^{\prime}}=\alpha_{A} \sqrt{k_{A}} \sum_{k_{1}=1}^{K} \mathbf{g}_{m k_{1}}\left(\sqrt{\tau p_{p} k_{U}} \boldsymbol{\varphi}_{k_{1}}^{H} \boldsymbol{\varphi}_{k^{\prime}}+\eta_{k_{1} t, k^{\prime}}\right)+$

$\alpha_{A} \sqrt{\tau p_{E} k_{A}} \mathbf{g}_{m E} \boldsymbol{\varphi}_{k}^{H} \boldsymbol{\varphi}_{k^{\prime}}+\alpha_{A} \boldsymbol{\eta}_{m r, k^{\prime}}+\alpha_{A} \mathbf{w}_{p m, k^{\prime}}+\tilde{\mathbf{w}}_{p m, k^{\prime}}$,

where $\eta_{k_{1} t, k^{\prime}}=\boldsymbol{\eta}_{k_{1} t}^{T} \boldsymbol{\varphi}_{k^{\prime}} \sim \mathcal{C N}\left(0, p_{p}\left(1-k_{U}\right)\right), \mathbf{w}_{p m, k^{\prime}}=$ $\mathbf{W}_{p m} \boldsymbol{\varphi}_{k^{\prime}}, \tilde{\mathbf{w}}_{p m, k^{\prime}}=\tilde{\mathbf{W}}_{p m} \boldsymbol{\varphi}_{k^{\prime}}$, the distribution of $\boldsymbol{\eta}_{m r, k^{\prime}}$ is shown as (9) at the top of the next page.

Theorem 1: Adopting the linear MMSE method at the $m$ th AP, the estimation of the channel vector $\mathbf{g}_{m k^{\prime}}$ can be given by

$$
\hat{\mathbf{g}}_{m k^{\prime}}=c_{m k^{\prime}} \tilde{\mathbf{y}}_{p m, k^{\prime}},
$$

where $c_{m k^{\prime}}$ can be given by (11) shown at the top of the next page.

In association with these results, the expectation of the norm-square of the channel estimate $\hat{\mathrm{g}}_{m k^{\prime}}$ and estimation error $\tilde{\mathrm{g}}_{m k^{\prime}}$ satisfy

$$
\begin{gathered}
\mathbb{E}\left\{\left\|\hat{\mathbf{g}}_{m k^{\prime}}\right\|^{2}\right\}=N \lambda_{m k^{\prime}}, \\
\mathbb{E}\left\{\left\|\tilde{\mathbf{g}}_{m k^{\prime}}\right\|^{2}\right\}=N\left(\beta_{m k^{\prime}}-\lambda_{m k^{\prime}}\right),
\end{gathered}
$$

where $\lambda_{m k^{\prime}}=\alpha_{A} \sqrt{k_{A} k_{U} \tau p_{p}} c_{m k^{\prime}} \beta_{m k^{\prime}}$.

Proof: Please see Appendix A.

Furthermore, since this paper considers the secure transmission issue, the normalized minimum square error (NMSE) of the channel estimation at the target user can be defined as

$$
\mathrm{NMSE}=\frac{\sum_{m=1}^{M} \mathbb{E}\left\{\left\|\tilde{\mathbf{g}}_{m k}\right\|^{2}\right\}}{\sum_{m=1}^{M} \mathbb{E}\left\{\left\|\mathbf{g}_{m k}\right\|^{2}\right\}}=\frac{\sum_{m=1}^{M}\left(\beta_{m k}-\lambda_{m k}\right)}{\sum_{m=1}^{M} \beta_{m k}} .
$$

Note that the accuracy of the channel estimation is monotonically increasing in ADC/DAC precision and the RF chain quality.

\section{SECRECY PERFORMANCE ANALYSIS}

In the downlink transmission phase, treating the achieved channel estimate as the true channel parameters, the $m$ th AP performs beamforming for data transmission. Let $s_{k_{1}}$ be the data intended for the $k_{1}$ th user with $\left|s_{k_{1}}\right|=1$, and $p_{d}$ be the average power constraint at each AP. Similar to some existing works [30], [31], using matched filter locally at each AP, the transmitted signal for all $K$ users at the $m$ th AP can be given by

$$
\mathbf{x}_{m}=\sum_{k_{1}=1}^{K} \sqrt{p_{d} k_{A} \gamma_{m k_{1}}} \hat{\mathbf{g}}_{m k_{1}}^{*} s_{k_{1}}+\boldsymbol{\eta}_{m, t},
$$

where $\gamma_{m k_{1}}$ denotes the power control coefficient corresponding to the power from the $m$ th AP to $k_{1}$ th user. Considering the power constraint on the $m$ th AP, the power control coefficients should satisfy

$$
\sum_{k_{1}=1}^{K} \gamma_{m k_{1}} \lambda_{m k_{1}} \leq \frac{1}{N}, m=1, \cdots, M .
$$

Besides, $k_{A}$ is the RF quality factor of the APs, $\boldsymbol{\eta}_{m, t}$ denotes the RF distortion noise at the $m$ th AP with distribution as (17) at the top of the next page.

As such, considering the RF impairments, the received signal at the $k$ th user and the Eve can be given by

$$
\begin{aligned}
r_{k}= & \sqrt{k_{U}} \sum_{m=1}^{M} \mathbf{g}_{m k}^{T}\left(\sum_{k_{1}=1}^{K} \sqrt{k_{A} \gamma_{m k_{1}} p_{d}} \hat{\mathbf{g}}_{m k_{1}}^{*} s_{k_{1}}+\boldsymbol{\eta}_{m, t}\right) \\
& +\eta_{k, r}+w_{k}, \\
r_{E}= & \sum_{m=1}^{M} \mathbf{g}_{m E}^{T}\left(\sum_{k_{1}=1}^{K} \sqrt{k_{A} \gamma_{m k_{1}} p_{d}} \hat{\mathbf{g}}_{m k_{1}}^{*} s_{k_{1}}+\boldsymbol{\eta}_{m, t}\right)+w_{E},
\end{aligned}
$$

where the term $\eta_{k, r}$ models the RF distortion noises at the $k$ th user whose statistical properties can be given by

$$
\eta_{k, r} \sim \mathcal{C N}\left(0,\left(1-k_{U}\right) \sum_{m=1}^{M} \sum_{k_{1}=1}^{K} \gamma_{m k_{1}} p_{d}\left|\mathbf{g}_{m k}^{T} \hat{\mathbf{g}}_{m k_{1}}^{*}\right|^{2}\right) .
$$

Besides, $w_{k} \sim \mathcal{C N}\left(0, \delta^{2}\right)$ and $w_{E} \sim \mathcal{C N}\left(0, \delta^{2}\right)$ are the AWGN at the $k$ th user and the Eve, respectively.

Furthermore, the output signal of the low resolution ADCs at the $k$ th user can be given by

$$
\begin{aligned}
\tilde{r}_{k}= & \alpha_{U} \sqrt{k_{U}} \sum_{m=1}^{M} \mathbf{g}_{m k}^{T}\left(\sum_{k_{1}=1}^{K} \sqrt{k_{A} \gamma_{m k_{1}} p_{d}} \hat{\mathbf{g}}_{m k_{1}}^{*} s_{k_{1}}+\boldsymbol{\eta}_{m, t}\right) \\
& +\alpha_{U} \eta_{k, r}+\alpha_{U} w_{k}+\tilde{w}_{k},
\end{aligned}
$$

where $\alpha_{U}$ represents the ADC quantization distortion factor of the legitimate users, $\tilde{w}_{k}$ is the additive Gaussian quantization noise with covariance as

$$
R_{\tilde{w}_{k}}=\mathbb{E}\left\{\left|\tilde{w}_{k}\right|^{2}\right\}=\alpha_{U}\left(1-\alpha_{U}\right) \mathbb{E}\left\{\left|r_{k}\right|^{2}\right\} .
$$

Then, we focus on the achievable closed-form capacity expressions of the target user and Eve. Furthermore, the ergodic rate is appropriate for the system performance evaluation and has been exploited in many existing works. This paper also utilizes the achievable ergodic secrecy rate for performance measure. Due to the RF chain distortion and coarse AD$\mathrm{Cs} / \mathrm{DACs}$, the channel estimation and terms at the right of (21) are non-Gaussian distributed which causes the standard capacity analysis impossible. Fortunately, the use-and-thenforget methodology can be used for capacity analysis. In particular, the received signal can be rewritten as

$$
\begin{aligned}
\tilde{r}_{k}= & D S_{k} \cdot s_{k}+B U_{k} \cdot s_{k}+\sum_{k^{\prime} \neq k}^{K} U I_{k k^{\prime}} \cdot s_{k^{\prime}}+H I_{t}^{A P} \\
& +H I_{r}^{U E}+A N_{k}+F N_{k},
\end{aligned}
$$

where

$$
\begin{aligned}
D S_{k} & =\mathbb{E}\left\{\alpha_{U} \sqrt{\rho_{d} k_{U} k_{A}} \sum_{m=1}^{M} \gamma_{m k}^{1 / 2} \hat{\mathbf{g}}_{m k}^{H} \mathbf{g}_{m k}\right\}, \\
B U_{k} & =\alpha_{U} \sqrt{\rho_{d} k_{U} k_{A}} \sum_{m=1}^{M} \gamma_{m k}^{1 / 2} \hat{\mathbf{g}}_{m k}^{H} \mathbf{g}_{m k}-D S_{k},
\end{aligned}
$$




$$
\boldsymbol{\eta}_{m r, k^{\prime}} \sim \mathcal{C N}\left(0,\left(1-k_{A}\right)\left(p_{p} \sum_{k_{1}=1}^{K} \operatorname{diag}\left(\left|\left[\mathbf{g}_{m k_{1}}\right]_{1}^{2}, \cdots,\left[\mathbf{g}_{m k_{1}}\right]_{N}^{2}\right|\right)+p_{E} \operatorname{diag}\left(\left|\left[\mathbf{g}_{m E}\right]_{1}^{2}, \cdots,\left[\mathbf{g}_{m E}\right]_{N}^{2}\right|\right)\right) \mathbf{I}_{\tau}\right)
$$

$$
c_{m k^{\prime}}=\frac{\sqrt{\tau \rho_{p} k_{A} k_{U}} \beta_{m k^{\prime}}}{\rho_{p} \sum_{k_{1}=1}^{K} \beta_{m k_{1}}\left(\tau k_{A} k_{U}\left|\boldsymbol{\varphi}_{k^{\prime}}^{H} \boldsymbol{\varphi}_{k_{1}}\right|^{2}+1-k_{A} k_{U}\right)+\left(\tau k_{A}\left|\boldsymbol{\varphi}_{k^{\prime}}^{H} \boldsymbol{\varphi}_{k}\right|^{2}+1-k_{A}\right) \rho_{E} \beta_{m E}+\delta^{2}}
$$

$$
\eta_{m, t} \sim \mathcal{C N}\left(\mathbf{0},\left(1-k_{A}\right) p_{d} \sum_{k_{1}=1}^{K} \gamma_{m k_{1}} \operatorname{diag}\left(\left|\left[\hat{\mathbf{g}}_{m k_{1}}\right]_{1}^{2}, \cdots,\left[\hat{\mathbf{g}}_{m k_{1}}\right]_{N}^{2}\right|\right)\right)
$$

$$
\begin{gathered}
U I_{k k^{\prime}}=\alpha_{U} \sqrt{\rho_{d} k_{U} k_{A}} \sum_{m=1}^{M} \gamma_{m k^{\prime}}^{1 / 2} \hat{\mathbf{g}}_{m k^{\prime}}^{H} \mathbf{g}_{m k}, \\
H I_{t}^{A P}=\alpha_{U} \sqrt{k_{U}} \sum_{m=1}^{M} \mathbf{g}_{m k}^{T} \eta_{m, t} \\
H I_{r}^{U E}=\alpha_{U} \eta_{k r} \\
A N_{k}=\alpha_{U} w_{k}, \\
F N_{k}=\tilde{w}_{k} .
\end{gathered}
$$

Also, it can be proved that the terms in (23) are pair-wisely uncorrelated. Then, we can derive the results as follows.

Theorem 2: Using the GRFIM and AQNM to model the imperfect RF chains and low-resolution ADCs/DACs, the expectations of the norm-square of the sum inner product and the norm-square of the Hadamard product of $\sqrt{\gamma_{m k^{\prime}}} \hat{\mathbf{g}}_{m k^{\prime}}$ and $\mathbf{g}_{m k}$ can be derived as

$$
\begin{aligned}
& \Omega_{k k^{\prime}}=\mathbb{E}\left\{\left|\sum_{m=1}^{M} \sqrt{\gamma_{m k^{\prime}}} \hat{\mathbf{g}}_{m k^{\prime}}^{H} \mathbf{g}_{m k}\right|^{2}\right\}= \\
& N^{2}\left(\frac{1-k_{U}}{\tau k_{U}}+\left|\boldsymbol{\varphi}_{k^{\prime}}^{H} \boldsymbol{\varphi}_{k}\right|^{2}\right)\left(\sum_{m=1}^{M} \sqrt{\gamma_{m k^{\prime}}} \lambda_{m k^{\prime}} \frac{\beta_{m k}}{\beta_{m k^{\prime}}}\right)^{2} \\
& +N \alpha_{A}^{2} \rho_{p}\left(1-k_{A}\right) \sum_{m=1}^{M} \gamma_{m k^{\prime}} c_{m k^{\prime}}^{2} \beta_{m k}^{2} \\
& +N \sum_{m=1}^{M} \gamma_{m k^{\prime}} \lambda_{m k^{\prime}} \beta_{m k}, \\
& \Psi_{k k^{\prime}}=\mathbb{E}\left\{\left|\sum_{m=1}^{M} \sqrt{\gamma_{m k^{\prime}}} \hat{\mathbf{g}}_{m k^{\prime}}^{H} \circ \mathbf{g}_{m k}\right|^{2}\right\}= \\
& N\left(\frac{1-k_{U}}{\tau k_{U}}+\left|\boldsymbol{\varphi}_{k^{\prime}}^{H} \boldsymbol{\varphi}_{k}\right|^{2}\right)\left(\sum_{m=1}^{M} \sqrt{\gamma_{m k^{\prime}}} \lambda_{m k^{\prime}} \frac{\beta_{m k}}{\beta_{m k^{\prime}}}\right)^{2} \\
& +N \alpha_{A}^{2} \rho_{p}\left(1-k_{A}\right) \sum_{m=1}^{M} \gamma_{m k^{\prime}} c_{m k^{\prime}}^{2} \beta_{m k}^{2} \\
& +N \sum_{m=1}^{M} \gamma_{m k^{\prime}} \lambda_{m k^{\prime}} \beta_{m k} .
\end{aligned}
$$

$$
r_{E}=D S_{E} \cdot s_{k}+\sum_{k^{\prime} \neq k}^{K} U I_{E, k k^{\prime}} \cdot s_{k^{\prime}}+H I_{E, t}^{A P}+w_{E},
$$

where

$$
D S_{E}=\sqrt{p_{d} k_{A}} \sum_{m=1}^{M} \gamma_{m k}^{1 / 2} \hat{\mathbf{g}}_{m k}^{H} \mathbf{g}_{m E}
$$




$$
R_{k}=\log _{2}\left(1+\frac{\left|D S_{k}\right|^{2}}{\mathbb{E}\left\{\left|B U_{k}\right|^{2}+\sum_{k^{\prime} \neq k}^{K}\left|U I_{k k^{\prime}}\right|^{2}+\left|H I_{t}^{A P}\right|^{2}+\left|H I_{r}^{U E}\right|^{2}+\left|A N_{k}\right|^{2}+\left|F N_{k}\right|^{2}\right\}}\right)
$$

$$
\begin{gathered}
U I_{E, k k^{\prime}}=\sqrt{p_{d} k_{A}} \sum_{m=1}^{M} \gamma_{m k^{\prime}}^{1 / 2} \hat{\mathbf{g}}_{m k^{\prime}}^{H} \mathbf{g}_{m E}, \\
H I_{E, t}^{A P}=\sum_{m=1}^{M} \eta_{m t}^{H} \mathbf{g}_{m E} .
\end{gathered}
$$

The first term in (41) is the desired signal, and the other terms are treated as aggregated noise. Also, it is proved that the terms $U I_{E, k k^{\prime}}, H I_{E, t}^{A P}$ and $w_{E}$ are pair-wisely uncorrelated. Thus, we can consider of the ergodic information capacity of the Eve as follows.

Theorem 3: Considering the case that both the APs and the legitimate users are equipped with low resolution ADCs/DACs and imperfect RF chains while the active Eve is with perfect hardware, the expectation of the norm-square of the sum inner product and the Hadamard product of $\sqrt{\gamma_{m k^{\prime}}} \hat{\mathbf{g}}_{m k^{\prime}}$ and $\mathbf{g}_{m E}$ are given by

$$
\begin{aligned}
& \Omega_{E k^{\prime}}=\mathbb{E}\left\{\left|\sum_{m=1}^{M} \sqrt{\gamma_{m k^{\prime}}} \hat{\mathbf{g}}_{m k^{\prime}}^{H} \mathbf{g}_{m E}\right|^{2}\right\} \\
& =N^{2} \alpha_{A}^{2} k_{A} \tau \rho_{E}\left|\boldsymbol{\varphi}_{k^{\prime}}^{H} \boldsymbol{\varphi}_{k}\right|^{2}\left(\sum_{m=1}^{M} \sqrt{\gamma_{m k^{\prime}}} c_{m k^{\prime}} \beta_{m E}\right)^{2} \\
& +N \alpha_{A}^{2} \rho_{E}\left(1-k_{A}\right)\left|\boldsymbol{\varphi}_{k^{\prime}}^{H} \boldsymbol{\varphi}_{k}\right|^{2} \sum_{m=1}^{M} \gamma_{m k^{\prime}} c_{m k^{\prime}}^{2} \beta_{m E}^{2} \\
& +N \sum_{m=1}^{M} \gamma_{m k^{\prime}} \lambda_{m k^{\prime}} \beta_{m E}, \\
& \Psi_{E k^{\prime}}=\mathbb{E}\left\{\left|\sum_{m=1}^{M} \sqrt{\gamma_{m k^{\prime}}} \hat{\mathbf{g}}_{m k^{\prime}}^{H} \circ \mathbf{g}_{m E}\right|^{2}\right\} \\
& =N \alpha_{A}^{2} k_{A} \tau \rho_{E}\left|\boldsymbol{\varphi}_{k^{\prime}}^{H} \boldsymbol{\varphi}_{k}\right|^{2}\left(\sum_{m=1}^{M} \sqrt{\gamma_{m k^{\prime}}} c_{m k^{\prime}} \beta_{m E}\right)^{2} \\
& +N \alpha_{A}^{2} \rho_{E}\left(1-k_{A}\right)\left|\boldsymbol{\varphi}_{k^{\prime}}^{H} \boldsymbol{\varphi}_{k}\right|^{2} \sum_{m=1}^{M} \gamma_{m k^{\prime}} c_{m k^{\prime}}^{2} \beta_{m E}^{2} \\
& +N \sum_{m=1}^{M} \gamma_{m k^{\prime}} \lambda_{m k^{\prime}} \beta_{m E} .
\end{aligned}
$$

Then, the upper-bound for the ergodic rate of the Eve can be formulated as

$R_{E}=\log _{2}\left(1+\frac{\mathbb{E}\left\{\left|D S_{E}\right|^{2}\right\}}{\mathbb{E}\left\{\sum_{k^{\prime} \neq k}^{K}\left|U I_{E, k k^{\prime}}\right|^{2}+\left|H I_{E, t}^{A P}\right|^{2}+\left|w_{E}\right|^{2}\right\}}\right)$ where

$$
\begin{aligned}
& \mathbb{E}\left\{\left|D S_{E}\right|^{2}\right\}=\mathbb{E}\left\{\left|\sqrt{\rho_{d} k_{A}} \sum_{m=1}^{M} \gamma_{m k}^{1 / 2} \hat{\mathbf{g}}_{m k}^{H} \mathbf{g}_{m E}\right|^{2}\right\} \\
&= \rho_{d} k_{A} \Omega_{E k}, \\
& \mathbb{E}\left\{\left|U I_{E, k k^{\prime}}\right|^{2}\right\}=\mathbb{E}\left\{\left|\sqrt{\rho_{d} k_{A}} \sum_{m=1}^{M} \gamma_{m k^{\prime}}^{1 / 2} \hat{\mathbf{g}}_{m k^{\prime}}^{H} \mathbf{g}_{m E}\right|^{2}\right\} \\
&= \rho_{d} k_{A} \Omega_{E k^{\prime}}, \\
& \mathbb{E}\left\{\left|H I_{E, t}^{A P}\right|^{2}\right\}=\rho_{d} k_{U}\left(1-k_{A}\right) \sum_{k^{\prime}=1}^{K} \Psi_{E k^{\prime}}, \\
& \mathbb{E}\left\{\left|w_{E}\right|^{2}\right\}=\delta^{2} .
\end{aligned}
$$

Proof: Please refer to Appendix C.

According to some existing papers [15], [19], [21], the ergodic secrecy rate can be used for secure performance analysis which is appropriate for the delay afford systems over many coherence intervals or many channel realizations. Note that there are no pre-log factors in (33) and (47). It can be explained that achievable values are linear to the real rate values (with pre-log factors) which also can be exploited to evaluate the system performance. In the end, the ergodic secrecy rate can be defined as follows:

$$
R_{\mathrm{sec}, k}=\left[R_{k}-R_{E}\right]^{+},
$$

where the closed-form expressions of $R_{k}$ and $R_{E}$ have been presented in (33) and (47).

The closed-form expression (52) of the achievable ergodic secrecy rate shows the impacts of the RF impairments and ADC/DAC imperfections. It it noted that the derived expression is consistent with the existing results with perfect RF chians or without active eavesdropping [21], [30]. In addition, it can be proved that the achievable ergodic secrecy rate is monotonically increasing in quantization distortion factor $\alpha_{A}\left(\alpha_{U}\right)$ and the RF quality factors $k_{A}\left(k_{U}\right)$ by computing the derivative of the secrecy rate $R_{\mathrm{sec}, k}$. To gain more insight, the compensations between the RF impairments and the resolution of $\mathrm{ADCs} / \mathrm{DACs}$ can be considered based on the achievable ergodic secrecy rate expression as (52). The compensations imply that the performance loss with low quality RF chains can be compensated by equipping high resolution $\mathrm{ADCs} / \mathrm{DACs}$, and vice versa. Unfortunately, due to the complexity of the expressions on channel estimation and the ergodic secrecy rate, it is implicit and challenging to quantify the compensation case and obtain some exact closedform solutions. In order to obtain useful insights, some special 
cases can be considered to investigate the compensations. To make the problem analysable, some assumptions can be made as follows: the APs and the legitimate user has the same quality of RF chains and ADCs/DACs, i.e. $\alpha_{A}=\alpha_{U}=\alpha$, $k_{A}=k_{U}=k$. In this case, the compensation issue can be solved by one-dimensional search method. To achieve some accurate compensation schemes, one iterative search algorithm can be designed as Algorithm 1.

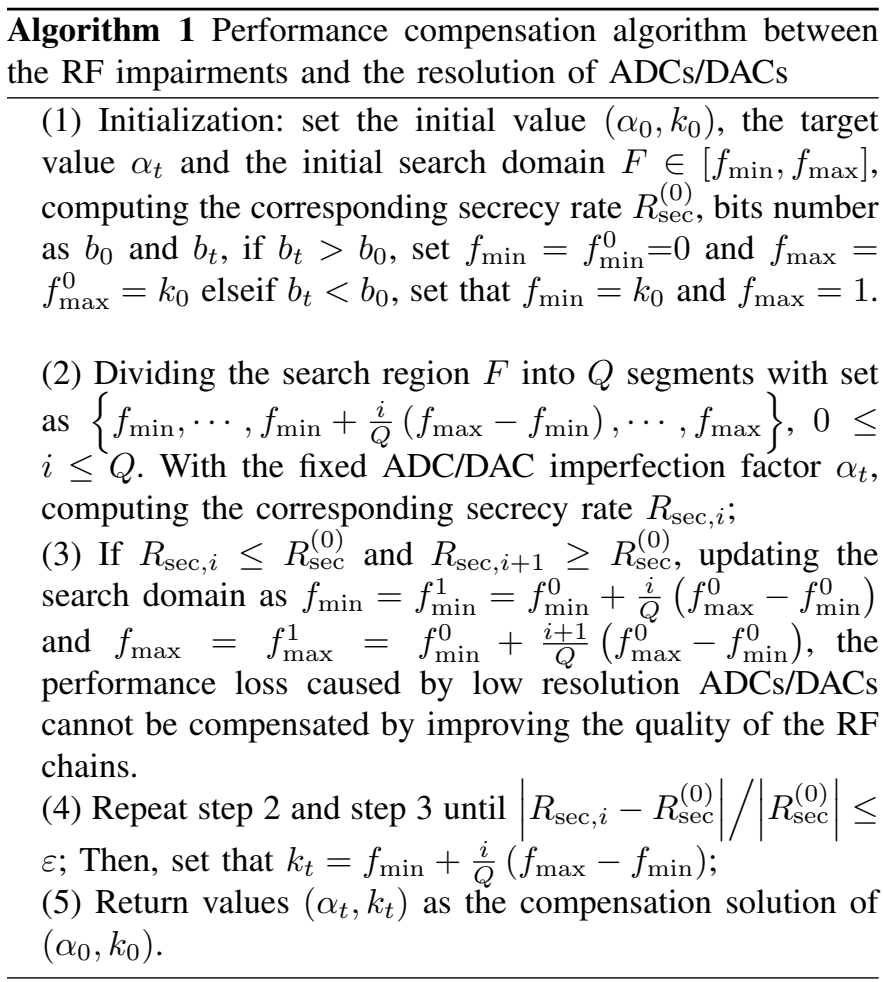

\section{Simulation Results}

In this section, representative simulation results are provided to evaluate the secrecy performance of the considered system. $M$ APs, $K$ legitimate users and the Eve are randomly uniformly distributed in a hexagonal cell region with a radius of $1000 \mathrm{~m}$. To investigate the pilot contamination issue, all sequences are non-orthogonal. For facilitating the analysis and design, different pilot sequences are designed orthogonally in the simulations while each pilot signal can be used by $K / \tau$ users. In addition, key system parameters in the simulations are set as follows: $M=200, K=10, N=2, \tau=5$, $p_{p}=100 \mathrm{~mW}, p_{E}=50 \mathrm{~mW}, p_{d}=100 \mathrm{~mW}$. The large-scale fading coefficient is composed of the path loss and the shadow fading which is modeled as

$$
\beta=P L \cdot 10^{\frac{\delta_{s h} z}{10}}
$$

where $P L$ denotes the path loss, $10 \frac{\delta_{s h} z}{10}$ represents the shadow fading with standard deviation $\delta_{s h}=8 \mathrm{~dB}$ and random variable $z \sim \mathcal{N}(0,1)$. According to some existing works [5], [7], [21],

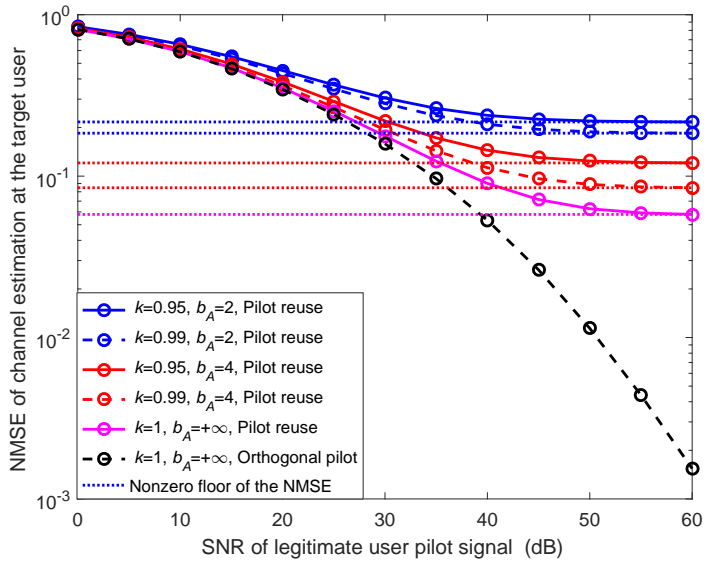

Fig. 3. The NMSE of the channel estimation at the target user versus SNR of the legitimate user pilot signal.

[22], [26], [31], the three-slope model is used to model the path loss, where the path loss model in $\mathrm{dB}$ is represented as

$$
P L=\left\{\begin{array}{c}
-L-35 \log _{10} d, \quad \text { if } d>d_{1} \\
-L-15 \log _{10} d_{1}-20 \log _{10} d, \text { if } d_{0}<d \leq d_{1} \\
-L-15 \log _{10} d_{1}-20 \log _{10} d_{0}, \text { if } d \leq d_{0}
\end{array}\right.
$$

where $L=140.7 \mathrm{~dB}, d_{0}=10 \mathrm{~m}, d_{1}=50 \mathrm{~m}$.

In accordance with the relevant literature, the noise power at the APs and all users in watt can be given by

$$
\delta^{2}=B \times k_{B} \times T_{0} \times \text { noise figure, }
$$

where $k_{B}=1.381 \times 10^{-23}(\mathrm{~J} /$ Kelvin $), T_{0}=290($ Kelvin $)$, $B=20 \mathrm{MHz}$, noise figure $=9 \mathrm{~dB}$.

Fig. 3 depicts the NMSE of the channel estimation versus the signal-to-noise ratio (SNR) of the legitimate user pilot signal with respect of the RF hardware quality coefficients $\left(k_{A}\right.$ and $\left.k_{U}\right), \mathrm{ADCs} / \mathrm{DACs}$ quantization factor $\alpha_{A}$ at the AP and the pilot attacking power $p_{E}$. To facilitate comparison and analysis, we firstly consider the situation that $k_{U}=k_{A}$ and $\alpha_{U}=\alpha_{A}$. It is noted that the NMSE degrades with the decrease of the transceivers quality, which implies that low resolution $\mathrm{ADCs} / \mathrm{DACs}$ and $\mathrm{RF}$ impairments deteriorate the accuracy of the linear MMSE channel estimation. Since the Eve sends the same pilot signals as the target user, it will degrade the pilot signal reception and increase the channel estimation uncertainty at the APs. Besides, the pilot reuse results in pilot contamination which will lead to a nonzero floor at the channel estimation error. Hence, it can be seen that there are nonzero estimation error floors in the high SNR regime (horizontal dashed lines in Fig. 3) caused by the RF distortions, low resolution ADCs/DACs and the pilot contamination. However, implementing perfect transceivers at the users and APs as well as the orthogonal pilots lead to an error-free channel estimation at high SNR.

Then, we present simulation results to verify the accuracy of the derived secrecy performance merits. Since it is difficult to accurately simulate the performance degradation caused by low resolution ADCs/DACs, the AQNM has been used in Monte-Carlo simulations to evaluate the simulated results. Fig. 4 shows the AQNM-based Monte Carlo simulated and 

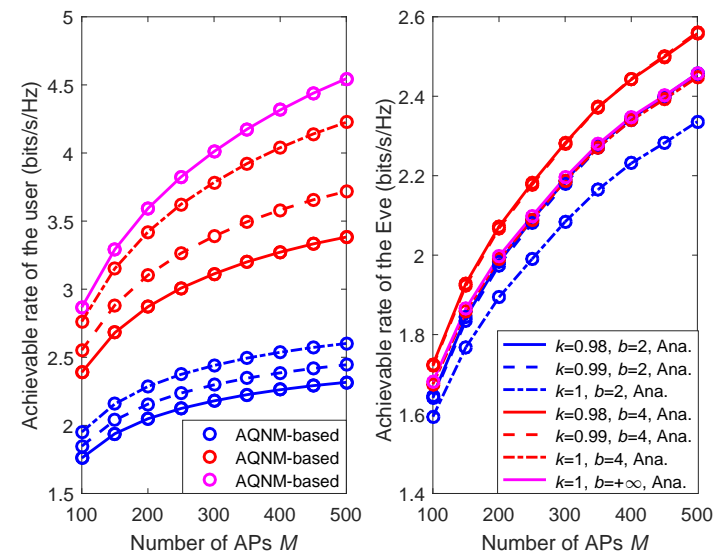

Fig. 4. Ergodic rates of the target user and the Eve versus the number of the APs $M$.

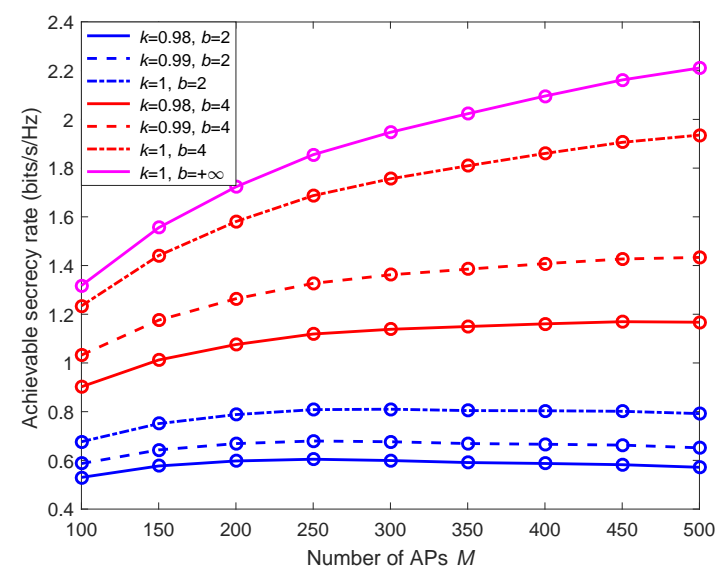

Fig. 5. The achievable ergodic secrecy rates versus the number of the APs M.

analytical results of the $R_{k}$ and $R_{E}$ versus $M$, where the simulated results are obtained by averaging over 5000 independent channel realizations. As expected, more APs yield improved coverage probability, which can be proved by the fact that the achievable ergodic rate of the target user and the ergodic capacity of the Eve are both monotonically increasing with the increase of $M$. Different from the passive eavesdropper which cannot get distinct benefits by increasing the total antennas [19], the achievable capacity of the active Eve takes advantage of equipping more antennas. It is gratifying to observe that the analytical results are tight with the simulated numerical values in high accuracy. In addition, worse transceiver RF chains or lower quality ADCs/DACs may bring about more information leakage. Thereby, perhaps the Eve could not achieve more information from perfect RF chains. Then, Fig. 5 shows the achievable secrecy rate versus $M$ with different quality of $\mathrm{ADC} / \mathrm{DAC}$ and RF chains. It is clear that the ergodic secrecy rate reduces as the quantization distortion factors of the $\mathrm{ADCs} / \mathrm{DACs}$ and the RF quality factors decrease. Besides, we note that the low quality ADCs/DACs and the RF impairments provoke a significant deterioration to the ergodic secrecy rate.

To clarify the effects of RF impairments and ADC/DAC

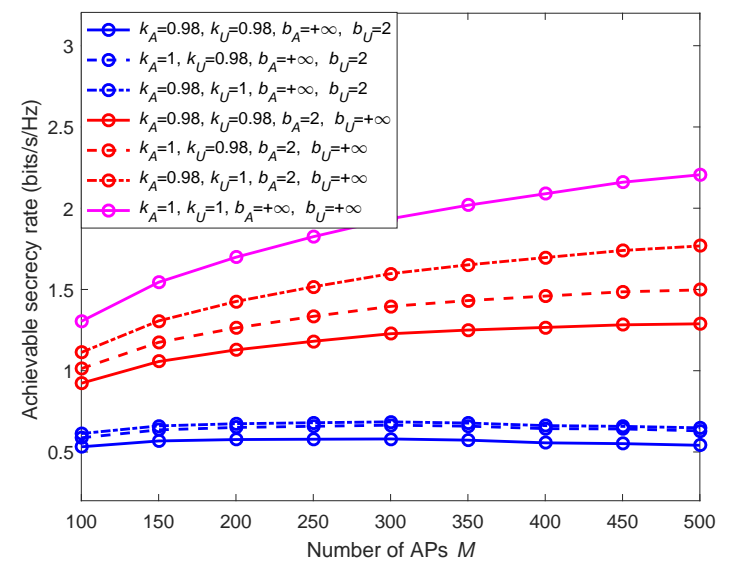

Fig. 6. The achievable ergodic secrecy rates with different hardware equipping schemes.

quality, the impacts of hardware quality on users and APs, are shown in Fig. 6 with different hardware equipping schemes (with different $k_{U}, k_{A}, \alpha_{U}$ and $\alpha_{A}$ ). It can be seen that the ergodic secrecy rate decreases with deploying low quality ADC/DAC and RF chains. Nevertheless, it is noted that the quality of the RF chain and ADCs at the legitimate user has a more serious impact on the ergodic secrecy rate since the ergodic secrecy rates with low quality hardware at the users are inferior to the case with the same quality factor at the APs. This observation means that equipping more APs can effectively compensate the performance loss caused by the low quality of APs while it cannot mitigate the detrimental effect of imperfect transceiver RF chains and coarse ADCs/DACs at the users.

Fig. 7 depicts the ergodic secrecy rate versus the quantization bits of low resolution ADCs with different quality of the RF chains. It is observed that increasing the ADCs/DACs quantization precision has a positive effect on the ergodic secrecy rate which can be explained by the fact that the secrecy rate is a monotonically increasing function of ADCs' quantization accuracy. Moreover, it can be noticed that the secrecy rate gradually converges to a fixed limit which is determined by the RF quality. Besides, when $b \geq 5$, the ergodic secrecy rate is extremely close to the finite limit values which is consistent with the conclusion in [26]. This observation indicates that equipping with 5-bit $\mathrm{ADCs} / \mathrm{DACs}$ leads to an insignificant capacity loss and this is effective enough for practical cell-free massive MIMO system.

Fig. 8 shows the compensation between ADCs/DACs quantization bits $b$ and RF quality factors $k$ where $b_{U}=b_{A}=b$ and $k_{U}=k_{A}=k$. The curves are obtained by using the proposed compensation algorithm against different achievable secrecy rate. In order to facilitate the comparison, each curve is calculated with initialization setting as $b=1$ and $k=1$. As it can be seen, with the increase of the values of $b$, the proposed compensation algorithm has been operated to determine the corresponding value of $k$. Each curve in Fig. 8 can achieve the same ergodic secrecy rate, that is, improving the precision of $\mathrm{ADCs} / \mathrm{DACs}$ can tolerate worse RF chain quality, and vice versa. Hence, these results are extremely 


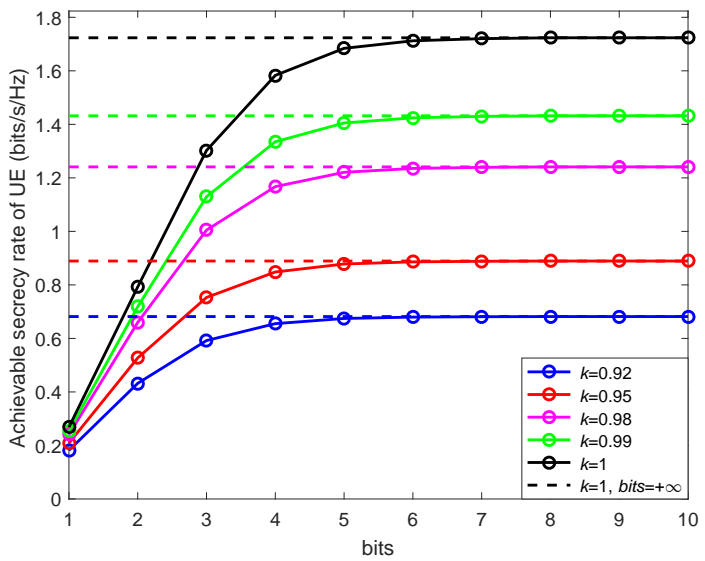

Fig. 7. The achievable ergodic secrecy rates versus the quantization bits of low resolution ADCs.

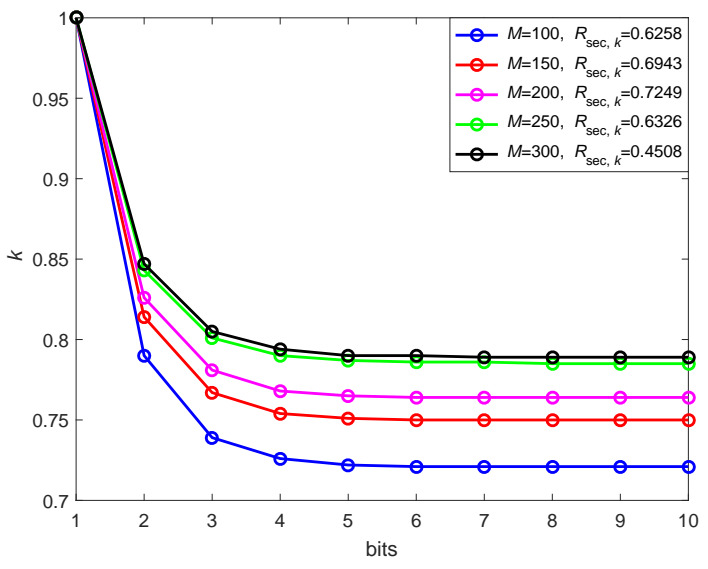

Fig. 8. Illustration of the compensation between ADCs resolution $b$ and RF quality factors $k$.

valuable and constructive for optimizing the hardware costs and energy efficient in practical system design.

\section{CONCLUSION}

This paper investigated the secure transmission in a cell-free massive MIMO system with RF impairments and low resolution $\mathrm{ADCs} / \mathrm{DACs}$ in the presence of an active eavesdropper. Using the AQNM and the GRFIM, closed-form expressions of linear MMSE channel estimation and achievable ergodic secrecy rate have been derived with respect to key system parameters, including pilot power, the number of legitimate users and the total antennas, $\mathrm{ADCs} / \mathrm{DACs}$ quality factors and transceiver RF impairment factors. It is noted that there is nonzero floor on the channel estimation error caused by the low-quality transceivers. Moreover, based on the achievable ergodic secrecy rate expression, the compensation algorithm between RF impairments and ADCs/DACs resolution has been presented in detail which is feasible for flexibly equipping coarse ADCs/DACs and economical RF components in practical system design.

\section{ACKNOWLEDGMENT}

This work was supported by the National Natural Science Foundation of China under Grant 61901502 and Grant
U19B214, in part by the UK Engineering and Physical Sciences Research Council (EPSRC) under the grant EP/N007840/1.

\section{APPENDIX}

\section{A. Proof of Theorem 1}

Based on (8), the post-processing signal $\tilde{\mathbf{y}}_{p m, k^{\prime}}$ can be rewritten as

$$
\begin{aligned}
& \tilde{\mathbf{y}}_{p m, k^{\prime}}=\alpha_{A} \sum_{k_{1} \in P_{k}} \sqrt{\tau p_{p} k_{A} k_{U}} \mathbf{g}_{m k_{1}}+ \\
& \alpha_{A} \sqrt{k_{A}} \sum_{k_{1}=1}^{K} \mathbf{g}_{m k_{1}} \eta_{k_{1} t, k^{\prime}}+\alpha_{A} \sqrt{\tau p_{E} k_{A}} \mathbf{g}_{m E} \boldsymbol{\varphi}_{k}^{H} \boldsymbol{\varphi}_{k^{\prime}} \\
& +\alpha_{A} \boldsymbol{\eta}_{m r, k^{\prime}}+\alpha_{A} \mathbf{w}_{p m, k^{\prime}}+\tilde{\mathbf{w}}_{p m, k^{\prime}} .
\end{aligned}
$$

It remarks that the different terms are mutually independent of each other and are all Gaussian RVs with zero mean. Based on the linear MMSE algorithm, we can obtain that

$$
\hat{\mathbf{g}}_{m k^{\prime}}=\frac{\mathbb{E}\left\{\tilde{\mathbf{y}}_{p m, k^{\prime}}^{H} \mathbf{g}_{m k^{\prime}}\right\}}{\mathbb{E}\left\{\tilde{\mathbf{y}}_{p m, k^{\prime}}^{H} \tilde{\mathbf{y}}_{p m, k^{\prime}}\right\}} \tilde{\mathbf{y}}_{p m, k^{\prime}},
$$

First, we focus on the term $\mathbb{E}\left\{\tilde{\mathbf{y}}_{p m, k^{\prime}}^{H} \mathbf{g}_{m k^{\prime}}\right\}$ as

$$
\mathbb{E}\left\{\tilde{\mathbf{y}}_{p m, k^{\prime}}^{H} \mathbf{g}_{m k^{\prime}}\right\}=N \alpha_{A} \sqrt{\tau p_{p} k_{A} k_{U}} \beta_{m k^{\prime}} .
$$

We make a definition that as

$$
\begin{aligned}
& \mathbf{y}_{p m, k^{\prime}}=\sum_{k_{1} \in P_{k}} \sqrt{\tau p_{p} k_{A} k_{U}} \mathbf{g}_{m k_{1}}+\sqrt{k_{A}} \sum_{k_{1}=1}^{K} \mathbf{g}_{m k_{1}} \eta_{k_{1} t, k^{\prime}} \\
& +\sqrt{\tau p_{E} k_{A}} \mathbf{g}_{m E} \varphi_{k}^{H} \varphi_{k^{\prime}}+\eta_{m r, k^{\prime}}+\mathbf{w}_{p m, k^{\prime}} .
\end{aligned}
$$

As such, we can recast (56) as

$$
\tilde{\mathbf{y}}_{p m, k^{\prime}}=\alpha_{A} \mathbf{y}_{p m, k^{\prime}}+\tilde{\mathbf{w}}_{p m, k^{\prime}} .
$$

The covariance of the quantization noise can be given by

$$
\mathbb{E}\left\{\mathbf{w}_{p m, k^{\prime}}^{H} \mathbf{w}_{p m, k^{\prime}}\right\}=\alpha_{A}\left(1-\alpha_{A}\right) \mathbb{E}\left\{\mathbf{y}_{p m, k^{\prime}}^{H} \mathbf{y}_{p m, k^{\prime}}\right\} .
$$

Hence, we can have that

$$
\begin{aligned}
\mathbb{E}\left\{\tilde{\mathbf{y}}_{p m, k^{\prime}}^{H} \tilde{\mathbf{y}}_{p m, k^{\prime}}\right\} & =\alpha_{A}^{2} \mathbb{E}\left\{\mathbf{y}_{p m, k^{\prime}}^{H} \mathbf{y}_{p m, k^{\prime}}\right\} \\
& +\alpha_{A}\left(1-\alpha_{A}\right) \mathbb{E}\left\{\mathbf{y}_{p m, k^{\prime}}^{H} \mathbf{y}_{p m, k^{\prime}}\right\} \\
& =\alpha_{A} \mathbb{E}\left\{\mathbf{y}_{p m, k^{\prime}}^{H} \mathbf{y}_{p m, k^{\prime}}\right\} .
\end{aligned}
$$

According to the definition and statistic properties of different terms in (59), we can derive that

$$
\begin{aligned}
& \mathbb{E}\left\{\mathbf{y}_{p m, k^{\prime}}^{H} \mathbf{y}_{p m, k^{\prime}}\right\}=\tau p_{p} k_{A} k_{U} \sum_{k_{1} \in P_{k}} \mathbb{E}\left\{\left\|\mathbf{g}_{m k_{1}}\right\|^{2}\right\}+ \\
& k_{A} \sum_{k_{1}=1}^{K} \mathbb{E}\left\{\left\|\mathbf{g}_{m k_{1}} \eta_{k_{1} t, k^{\prime}}\right\|^{2}\right\}+\tau p_{E} k_{A}\left|\boldsymbol{\varphi}_{k}^{H} \boldsymbol{\varphi}_{k^{\prime}}\right|^{2} \mathbb{E}\left\{\left\|\mathbf{g}_{m E}\right\|^{2}\right\} \\
& +\mathbb{E}\left\{\left\|\eta_{m r, k^{\prime}}\right\|^{2}\right\}+\mathbb{E}\left\{\left\|\mathbf{w}_{p m, k^{\prime}}\right\|^{2}\right\} \\
& =N \rho_{p} \sum_{k_{1}=1}^{K} \beta_{m k_{1}}\left(\tau k_{A} k_{U}\left|\boldsymbol{\varphi}_{k^{\prime}}^{H} \boldsymbol{\varphi}_{k_{1}}\right|^{2}+1-k_{A} k_{U}\right) \\
& +\left(\tau k_{A}\left|\boldsymbol{\varphi}_{k^{\prime}}^{H} \boldsymbol{\varphi}_{k}\right|^{2}+1-k_{A}\right) \rho_{E} \beta_{m E}+\delta^{2}
\end{aligned}
$$


Plugging the expressions of (58) and (62) into (57), we can get the expression of $c_{m k^{\prime}}$ as (11).

Then, the expectation of the norm-square of the channel estimation can be obtained as

$$
\begin{aligned}
\mathbb{E}\left\{\hat{\mathbf{g}}_{m k^{\prime}}^{H} \hat{\mathbf{g}}_{m k^{\prime}}\right\} & =c_{m k^{\prime}}^{2} \mathbb{E}\left\{\tilde{\mathbf{y}}_{p m, k^{\prime}}^{H} \tilde{\mathbf{y}}_{p m, k^{\prime}}\right\} \\
& =N \alpha_{A} \sqrt{k_{A} k_{U} \tau p_{p}} c_{m k^{\prime}} \beta_{m k^{\prime}} \\
& =N \lambda_{m k^{\prime}} .
\end{aligned}
$$

Besides, the estimation and estimation error are uncorrelated, i.e. $\mathbb{E}\left\{\tilde{\mathbf{g}}_{m k^{\prime}}^{H} \hat{\mathbf{g}}_{m k^{\prime}}\right\}=0$. Furthermore, we can calculate that

$$
\begin{aligned}
\mathbb{E}\left\{\tilde{\mathbf{g}}_{m k^{\prime}}^{H} \tilde{\mathbf{g}}_{m k^{\prime}}\right\} & =\mathbb{E}\left\{\mathbf{g}_{m k^{\prime}}^{H} \mathbf{g}_{m k^{\prime}}\right\}-\mathbb{E}\left\{\hat{\mathbf{g}}_{m k^{\prime}}^{H} \hat{\mathbf{g}}_{m k^{\prime}}\right\} \\
& =N\left(\beta_{m k^{\prime}}-\lambda_{m k^{\prime}}\right) .
\end{aligned}
$$

Thus, the proof is completed.

\section{B. Proof of Theorem 2}

Firstly, based on the uncorrelated characters of $\hat{\mathbf{g}}_{m k}$ and $\tilde{\mathbf{g}}_{m k}$ along with the used GRFIM and AQNM, desired results can be obtained as follows:

$$
\begin{gathered}
D S_{k}=\mathbb{E}\left\{\alpha_{U} \sqrt{\rho_{d} k_{U} k_{A}} \sum_{m=1}^{M} \gamma_{m k}^{1 / 2} \hat{\mathbf{g}}_{m k}^{H}\left(\hat{\mathbf{g}}_{m k}+\tilde{\mathbf{g}}_{m k}\right)\right\} \\
=N \alpha_{U} \sqrt{\rho_{d} k_{U} k_{A}} \sum_{m=1}^{M} \gamma_{m k}^{1 / 2} \lambda_{m k} \\
\mathbb{E}\left\{\left|B U_{k}\right|^{2}\right\}=\alpha_{U}^{2} \rho_{d} k_{U} k_{A} \Omega_{k k}-\left|D S_{k}\right|^{2} \\
\mathbb{E}\left\{\left|U I_{k k^{\prime}}\right|^{2}\right\}=\alpha_{U}^{2} \rho_{d} k_{U} k_{A} \Omega_{k k^{\prime}}, \\
\mathbb{E}\left\{\left|H I_{t}^{A P}\right|^{2}\right\}=\alpha_{U}^{2} \rho_{d} k_{U}\left(1-k_{A}\right) \sum_{k^{\prime}=1}^{K} \Psi_{k k^{\prime}} \\
\mathbb{E}\left\{\left|F N_{k}\right|^{2}\right\}=\alpha_{U}^{2}\left(1-\alpha_{U}\right) \cdot \\
M \rho_{d}\left(1-k_{U}\right) \cdot \\
\left(k_{A} \rho_{d} \sum_{m=1}^{K} \Omega_{k k^{\prime}}+\left(1-k_{A}\right) \rho_{d} \sum_{m=1}^{M} \Psi_{k k^{\prime}}+\delta^{2}\right) \\
\left.k_{A} \sum_{k^{\prime}=1} \Omega_{k k^{\prime}}+\left(1-k_{A}\right) \sum_{k^{\prime}=1}^{K} \Psi_{k k^{\prime}}\right)
\end{gathered}
$$

Then, the term $\Omega_{k k^{\prime}}$ can be rewritten as

$$
\begin{aligned}
\Omega_{k k^{\prime}} & =\mathbb{E}\left\{\left|\sum_{m=1}^{M} \sqrt{\gamma_{m k^{\prime}}} \hat{\mathbf{g}}_{m k^{\prime}}^{H} \mathbf{g}_{m k}\right|^{2}\right\} \\
& =\mathbb{E}\left\{\left|\sum_{m=1}^{M} c_{m k^{\prime}} \sqrt{\gamma_{m k^{\prime}}} \tilde{\mathbf{y}}_{p m, k^{\prime}}^{H} \mathbf{g}_{m k}\right|^{2}\right\} \\
& =\zeta_{1}+\zeta_{2}+\zeta_{3}+\zeta_{4}+\zeta_{5}+\zeta_{6},
\end{aligned}
$$

where

$\zeta_{1}=$

$\mathbb{E}\left\{\left|\sum_{m=1}^{M} \alpha_{A} c_{m k^{\prime}} \sqrt{\gamma_{m k^{\prime}} \tau p_{p} k_{U} k_{A}} \sum_{k_{1}=1}^{K} \mathbf{g}_{m k_{1}}^{H} \mathbf{g}_{m k} \boldsymbol{\varphi}_{k_{1}}^{H} \boldsymbol{\varphi}_{k^{\prime}}\right|^{2}\right\}$,

$$
\zeta_{2}=\mathbb{E}\left\{\left|\sum_{m=1}^{M} \alpha_{A} c_{m k^{\prime}} \sqrt{\gamma_{m k^{\prime}} k_{A}} \sum_{k_{1}=1}^{K} \eta_{k_{1} t, k^{\prime}} \mathbf{g}_{m k_{1}}^{H} \mathbf{g}_{m k}\right|^{2}\right\}
$$

$$
\zeta_{3}=\mathbb{E}\left\{\left|\sum_{m=1}^{M} \alpha_{A} c_{m k^{\prime}} \sqrt{\gamma_{m k^{\prime}} \tau p_{E} k_{A}} \mathbf{g}_{m E}^{H} \mathbf{g}_{m k} \boldsymbol{\varphi}_{k}^{H} \boldsymbol{\varphi}_{k^{\prime}}\right|^{2}\right\}
$$

$$
\begin{aligned}
& \zeta_{4}=\mathbb{E}\left\{\left|\sum_{m=1}^{M} \sqrt{\gamma_{m k^{\prime}}} \alpha_{A} c_{m k^{\prime}} \boldsymbol{\eta}_{m r, k^{\prime}}^{H} \mathbf{g}_{m k}\right|^{2}\right\}, \\
& \zeta_{5}=\mathbb{E}\left\{\left|\sum_{m=1}^{M} \sqrt{\gamma_{m k^{\prime}}} \alpha_{A} c_{m k^{\prime}} \mathbf{w}_{p m, k^{\prime}}^{H} \mathbf{g}_{m k}\right|^{2}\right\},
\end{aligned}
$$

$$
\zeta_{6}=\mathbb{E}\left\{\left|\sum_{m=1}^{M} \sqrt{\gamma_{m k^{\prime}}} c_{m k^{\prime}} \tilde{\mathbf{w}}_{p m, k^{\prime}}^{H} \mathbf{g}_{m k}\right|^{2}\right\}
$$

Then, some algebraic manipulations should be presented to compute the above terms respectively as

$$
\begin{aligned}
& \zeta_{1}=\alpha_{A}^{2} \tau p_{p} k_{U} k_{A}\left(\mathbb{E}\left\{\left|\sum_{m=1}^{M} c_{m k^{\prime}} \sqrt{\gamma_{m k^{\prime}}} \mathbf{g}_{m k}^{H} \mathbf{g}_{m k} \boldsymbol{\varphi}_{k}^{H} \boldsymbol{\varphi}_{k^{\prime}}\right|^{2}\right\}\right. \\
& \left.+\mathbb{E}\left\{\left|\sum_{m=1}^{M} c_{m k^{\prime}} \sqrt{\gamma_{m k^{\prime}}} \sum_{k_{1} \neq k}^{K} \mathbf{g}_{m k_{1}}^{H} \mathbf{g}_{m k} \boldsymbol{\varphi}_{k_{1}}^{H} \boldsymbol{\varphi}_{k^{\prime}}\right|^{2}\right\}\right) \\
& =\alpha_{A}^{2} \tau p_{p} k_{U} k_{A}\left(N^{2}\left(\sum_{m=1}^{M} c_{m k^{\prime}} \sqrt{\gamma_{m k^{\prime}}} \beta_{m k}\right)^{2}\right. \\
& \left.+N \sum_{m=1}^{M} c_{m k^{\prime}}^{2} \gamma_{m k^{\prime}} \beta_{m k}^{2}\right) \cdot\left|\boldsymbol{\varphi}_{k}^{H} \boldsymbol{\varphi}_{k^{\prime}}\right|^{2}+ \\
& N \alpha_{A}^{2} \tau p_{p} k_{U} k_{A} N \sum_{m=1}^{M} \sum_{k_{1} \neq k}^{K} c_{m k^{\prime}}^{2} \gamma_{m k^{\prime}} \beta_{m k_{1}} \beta_{m k} \cdot\left|\boldsymbol{\varphi}_{k_{1}}^{H} \boldsymbol{\varphi}_{k^{\prime}}\right|^{2} \\
& =\alpha_{A}^{2} \tau p_{p} k_{U} k_{A} \cdot\left(N^{2}\left(\sum_{m=1}^{M} c_{m k^{\prime}} \sqrt{\gamma_{m k^{\prime}}} \beta_{m k}\right)^{2} \cdot\left|\boldsymbol{\varphi}_{k}^{H} \boldsymbol{\varphi}_{k^{\prime}}\right|^{2}\right. \\
& \left.+N \sum_{m=1}^{M} \sum_{k_{1}=1}^{K} c_{m k^{\prime}}^{2} \gamma_{m k^{\prime}} \beta_{m k_{1}} \beta_{m k} \cdot\left|\boldsymbol{\varphi}_{k_{1}}^{H} \boldsymbol{\varphi}_{k^{\prime}}\right|^{2}\right)
\end{aligned}
$$




$$
\begin{gathered}
\zeta_{2}=k_{A} \alpha_{A}^{2} \mathbb{E}\left\{\left|\sum_{m=1}^{M} c_{m k^{\prime}} \sqrt{\gamma_{m k^{\prime}}}\left\|\mathbf{g}_{m k}\right\|^{2} \eta_{k_{1} t, k}\right|^{2}\right\}+ \\
k_{A} \alpha_{A}^{2} \mathbb{E}\left\{\left|\sum_{m=1}^{M} c_{m k^{\prime}} \sqrt{\gamma_{m k^{\prime}}} \sum_{k_{1} \neq k}^{K} \eta_{k_{1} t, k^{\prime}} \mathbf{g}_{m k_{1}}^{H} \mathbf{g}_{m k}\right|^{2}\right\} \\
=k_{A}\left(1-k_{U}\right) \alpha_{A}^{2}\left(N^{2}\left(\sum_{m=1}^{M} c_{m k^{\prime}}^{2} \gamma_{m k^{\prime}} \beta_{m k}\right)^{2}\right. \\
\left.+N \sum_{m=1}^{M} c_{m k^{\prime}} \sqrt{\gamma_{m k^{\prime}}} \sum_{k_{1}=1}^{K} c_{m k^{\prime}}^{2} \gamma_{m k^{\prime}} \beta_{m k_{1}} \beta_{m k}\right), \\
\zeta_{3}=\alpha_{A}^{2} \tau p_{E} k_{A} \sum_{m=1}^{M} c_{m k^{\prime}}^{2} \gamma_{m k^{\prime}} \beta_{m E} \beta_{m k} \cdot\left|\varphi_{k}^{H} \varphi_{k^{\prime}}\right|^{2}, \\
\zeta_{4}=N \alpha_{A}^{2}\left(1-k_{A}\right) \cdot \\
\sum_{m=1}^{M} c_{m k^{\prime}}^{2} \gamma_{m k^{\prime}}\left(p_{p} \beta_{m k}^{2}+p_{p} \sum_{k_{1}=1}^{K} \beta_{m k_{1}} \beta_{m k}+p_{E} \beta_{m E} \beta_{m k}\right) \\
\zeta_{6}=N \alpha_{A}\left(1-\alpha_{A}\right) \sum_{m=1}^{M} \frac{\alpha_{A}^{2} \delta^{2} \sum_{m=1}^{M} c_{m k^{\prime}}^{2} \gamma_{m k^{\prime}} \beta_{m k},}{\zeta_{m k} \beta_{m k^{\prime}} \beta_{m} \sqrt{\tau \rho_{p} k_{A} k_{U}}} \\
c_{m k^{\prime}}
\end{gathered}
$$

Now, plugging these results into (73), we can get the analytical result in (31) with some linear algebraic manipulations. Besides, utilizing the similar methodology, the expression of (32) can be achieved by some algebraic operations. Finally, by substituting the expressions of $\Omega_{k k^{\prime}}$ and $\Psi_{k k^{\prime}}$ into (36), (37), (38) and (40), the desired closed-form results of (33) as well as the ergodic rate of the $k$ th user can be obtained. This finishes the proof.

\section{Proof of Theorem 3}

In order to obtain the closed-form expression of the ergodic capacity of the Eve, it is noted that it is crucial to calculate the terms of $\Omega_{E k^{\prime}}$ and $\Psi_{E k^{\prime}}$. Now, we can rewrite $\Omega_{E k^{\prime}}$ as

$$
\begin{aligned}
\Omega_{E k^{\prime}} & =\mathbb{E}\left\{\left|\sum_{m=1}^{M} c_{m k^{\prime}} \sqrt{\gamma_{m k^{\prime}}} \tilde{\mathbf{y}}_{p m, k^{\prime}}^{H} \mathbf{g}_{m E}\right|^{2}\right\} \\
& =\varepsilon_{1}+\varepsilon_{2}+\varepsilon_{3}+\varepsilon_{4}+\varepsilon_{5}+\varepsilon_{6},
\end{aligned}
$$

where

$$
\begin{aligned}
& \varepsilon_{1}= \\
& \mathbb{E}\left\{\left|\sum_{m=1}^{M} \alpha_{A} c_{m k^{\prime}} \sqrt{\gamma_{m k^{\prime}} \tau p_{p} k_{U} k_{A}} \sum_{k_{1}=1}^{K} \mathbf{g}_{m k_{1}}^{H} \mathbf{g}_{m E} \boldsymbol{\varphi}_{k_{1}}^{H} \boldsymbol{\varphi}_{k^{\prime}}\right|^{2}\right\},
\end{aligned}
$$

$$
\begin{gathered}
\varepsilon_{2}= \\
\mathbb{E}\left\{\left|\sum_{m=1}^{M} \alpha_{A} c_{m k^{\prime}} \sqrt{\gamma_{m k^{\prime}} k_{A}} \sum_{k_{1}=1}^{K} \eta_{k_{1} t, k^{\prime}} \mathbf{g}_{m k_{1}}^{H} \mathbf{g}_{m E}\right|^{2}\right\}, \\
\varepsilon_{3}= \\
\mathbb{E}\left\{\left|\sum_{m=1}^{M} \alpha_{A} c_{m k^{\prime}} \sqrt{\gamma_{m k^{\prime}} \tau p_{E} k_{A}} \mathbf{g}_{m E}^{H} \mathbf{g}_{m E} \boldsymbol{\varphi}_{k}^{H} \boldsymbol{\varphi}_{k^{\prime}}\right|^{2}\right\}, \\
\varepsilon_{4}=\mathbb{E}\left\{\left|\sum_{m=1}^{M} \alpha_{A} c_{m k^{\prime}} \eta_{m r, k^{\prime}}^{H} \mathbf{g}_{m E}\right|^{2}\right\}, \\
\varepsilon_{5}=\mathbb{E}\left\{\left|\sum_{m=1}^{M} \alpha_{A} c_{m k^{\prime}} \mathbf{w}_{p m, k^{\prime}}^{H} \mathbf{g}_{m E}\right|^{2}\right\}, \\
\varepsilon_{6}=\mathbb{E}\left\{\left|\sum_{m=1}^{M} c_{m k^{\prime}} \tilde{\mathbf{w}}_{p m, k^{\prime}}^{H} \mathbf{g}_{m E}\right|^{2}\right\} .
\end{gathered}
$$

Due to the mutual independence between $\mathbf{g}_{m E}$ and $\mathbf{g}_{m i}(1 \leq i \leq K)$, the terms in (86) can be computed by

$$
\begin{gathered}
\varepsilon_{1}= \\
N \alpha_{A}^{2} \tau p_{p} k_{U} k_{A} \sum_{m=1}^{M} \sum_{k_{1}=1}^{K} c_{m k^{\prime}}^{2} \gamma_{m k^{\prime}} \beta_{m k_{1}} \cdot\left|\boldsymbol{\varphi}_{k_{1}}^{H} \boldsymbol{\varphi}_{k^{\prime}}\right|^{2}, \\
\varepsilon_{2}=N \alpha_{A}^{2} p_{p} k_{A}\left(1-k_{U}\right) \cdot \\
\sum_{m=1}^{M} \sqrt{k_{A}} \sum_{k_{1}=1}^{K} c_{m k^{\prime}}^{2} \gamma_{m k^{\prime}} \beta_{m k_{1}} \beta_{m E}, \\
\varepsilon_{3}=N^{2} \alpha_{A}^{2} k_{A} \tau p_{E}\left|\boldsymbol{\varphi}_{k^{\prime}}^{H} \boldsymbol{\varphi}_{k}\right|^{2}\left(\sum_{m=1}^{M} c_{m k^{\prime}} \sqrt{\gamma_{m k^{\prime}}} \beta_{m E}\right)^{2} \\
+N \alpha_{A}^{2} k_{A} \tau p_{E}\left|\boldsymbol{\varphi}_{k^{\prime}}^{H} \boldsymbol{\varphi}_{k}\right|^{2} \sum_{m=1}^{M} c_{m k^{\prime}}^{2} \gamma_{m k^{\prime}} \beta_{m E}^{2}, \\
\varepsilon_{4}=\mathbb{E}\left\{\left|\sum_{m=1}^{M} \alpha_{A} c_{m k^{\prime}} \eta_{m r, k^{\prime}}^{H} \mathbf{g}_{m E}\right|^{2}\right\} \\
=N \alpha_{A}^{2}\left(1-k_{A}\right) \cdot \\
\sum_{m=1}^{M} c_{m k^{\prime}}^{2}\left(\sum_{k_{1}=1}^{K} p_{p} \beta_{m k_{1}} \beta_{m E}+2 p_{E} \beta_{m E}^{2}\right), \\
\varepsilon_{5}=N \alpha_{A}^{2} \sum_{m=1}^{M} c_{m k^{\prime}}^{2} \delta^{2} \beta_{m E}, \\
\varepsilon_{6}=N \alpha_{A}\left(1-\alpha_{A}\right) \cdot \\
\sum_{m=1}^{M} \frac{c_{m k^{\prime}}^{2} \gamma_{m k^{\prime}} \beta_{m E} \beta_{m k^{\prime}} \sqrt{\tau \rho_{p} k_{A} k_{U}}}{c_{m k^{\prime}}} \\
=N\left(1-\alpha_{A}\right) \sum_{m=1}^{M} \gamma_{m k^{\prime}} \lambda_{m k^{\prime}} \beta_{m E} .
\end{gathered}
$$

Then, substituting the above six terms into (86), we can get the result as (45). With the same algebraic manipulations, the expressions of (46) and (47) as well as the ergodic capacity of the Eve can be derived, which can complete the proof. 


\section{REFERENCES}

[1] T. L. Marzetta, "Massive MIMO: an introduction," Bell Labs Techn. J., vol. 20, no. 2015, pp. 11-20, Mar. 2015

[2] E. G. Larsson, O. Edfors, F. Tufvesson, and T. L. Marzetta, "Massive MIMO for next generation wireless systems," IEEE Commun. Mag., vol. 52, no. 2, pp. 186-195, Feb. 2014.

[3] J. Zhang, C. Wen, S. Jin, X. Gao, and K. Wong, "On Capacity of Large-Scale MIMO Multiple Access Channels with Distributed Sets of Correlated Antennas," IEEE J. Sel. Areas Commun., vol. 31, no. 2, pp. 133-148, Feb. 2013.

[4] A. Yang, Y. Jing, C. Xing, Z. Fei, and J. Kuang, "Performance Analysis and Location Optimization for Massive MIMO Systems With Circularly Distributed Antennas," IEEE Trans. Wireless Commun., vol. 14, no. 10, pp. 5659-5671, Oct. 2015.

[5] H. Q. Ngo, A. Ashikhmin, H. Yang, E. G. Larsson, and T. L. Marzetta, "cell-free Massive MIMO Versus Small Cells," IEEE Trans. Wireless Commun., vol. 16, no. 3, pp. 1834-1850, Mar. 2017.

[6] H. Q. Ngo, L. Tran, T. Q. Duong, M. Matthaiou, and E. G. Larsson, "On the Total Energy Efficiency of cell-free Massive MIMO," IEEE Trans. Green Commun. Netw., vol. 2, no. 1, pp. 25-39, Mar. 2018.

[7] A. Papazafeiropoulos, P. Kourtessis, M. D. Renzo, S. Chatzinotas, and J. M. Senior, "Performance Analysis of Cell-Free Massive MIMO Systems: A Stochastic Geometry Approach," IEEE Trans. Veh. Technol., vol. 69, no. 4, pp. 3523-3537, Apr. 2020.

[8] H. V. Nguyen, V. Nguyen, O. A. Dobre, et al., "On the Spectral and Energy Efficiencies of Full-Duplex Cell-Free Massive MIMO," IEEE J. Sel. Areas Commun., vol. 38, no. 8, pp. 1698-1718, Aug. 2020.

[9] A. K. Papazafeiropoulos, P. Kourtessis, S. Chatzinotas, and J. M. Senior, "Multipair Two-Way DF Relaying With Cell-Free Massive MIMO," IEEE Open J. Commun. Society, vol. 2, pp. 423-438, Mar. 2021.

[10] A. Papazafeiropoulos, H. Q. Ngo, P. Kourtessis, S. Chatzinotas, and J. M. Senior, "Towards Optimal Energy Efficiency in Cell-Free Massive MIMO Systems," IEEE Trans. Green Commun. Netw., [Online] doi:10.1109/TGCN.2021.3059206.

[11] N. Yang, L. Wang, G. Geraci, M. Elkashlan, J. Yuan and M. Di Renzo, "Safeguarding 5G wireless communication networks using physical layer security," IEEE Commun. Mag., vol. 53, no. 4, pp. 20-27, Apr. 2015.

[12] Y. P. Hong, P. Lan, and C. J. Kuo, "Enhancing Physical-Layer Secrecy in Multiantenna Wireless Systems: An Overview of Signal Processing Approaches," IEEE Signal Process. Mag., vol. 30, no. 5, pp. 29-40, Sept. 2013.

[13] D. Kapetanovic, G. Zheng, and F. Rusek, "Physical layer security for massive MIMO: An overview on passive eavesdropping and active attacks," IEEE Commun. Mag., vol. 53, no. 6, pp. 21-27, Jun. 2015.

[14] Y. Wu, R. Schober, D. W. K. Ng, C. Xiao, and G. Caire, "Secure Massive MIMO Transmission With an Active Eavesdropper," IEEE Trans. Inf. Theory, vol. 62, no. 7, pp. 3880-3900, Jul. 2016.

[15] J. Zhu, R. Schober, and V. K. Bhargava, "Secure Transmission in Multicell Massive MIMO Systems," IEEE Trans. Wireless Commun., vol. 13, no. 9, pp. 4766-4781, Sept. 2014.

[16] Y. Wu, R. Schober, D. W. K. Ng, C. Xiao, and G. Caire, "Secure Massive MIMO Transmission With an Active Eavesdropper," IEEE Trans. Inf. Theory, vol. 62, no. 7, pp. 3880-3900, Jul. 2016.

[17] X. Zhang, D. Guo, K. Guo, and H. Niu, "Secure performance analysis and detection of pilot attack in massive multiple-input multiple-output system," Int. J. Distrib. Sensor Netw., vol. 14, no. 5, May 2018.

[18] X. Zhang, D. Guo, K. An, X. Liang, and W. Ma, "Secure Transmission in Multi-Pair AF Relaying Massive MIMO Networks Against Active Pilot Spoofing Attacks," IEEE Access, vol. 7, pp. 3547-3560, Jan. 2019.

[19] K. Guo, Y. Guo, and G. Ascheid, "Security-Constrained Power Allocation in MU-Massive-MIMO With Distributed Antennas," IEEE Trans. Wireless Commun., vol. 15, no. 12, pp. 8139-8153, Dec. 2016.

[20] X. Zhang, D. Guo, K. An, W. Ma, and K. Guo, "Secure transmission and power allocation in multiuser distributed massive MIMO systems," Wireless Netw., vol. 26, pp. 941-954, Feb. 2020.

[21] T. M. Hoang, H. Q. Ngo, T. Q. Duong, H. D. Tuan, and A. Marshall, "cell-free Massive MIMO Networks: Optimal Power Control Against Active Eavesdropping," IEEE Trans. Commun., vol. 66, no. 10, pp. 47244737, Oct. 2018.

[22] X. Zhang, D. Guo, K. An, Z. Ding, and B. Zhang, "Secrecy Analysis and Active Pilot Spoofing Attack Detection for Multigroup Multicasting cellfree Massive MIMO Systems," IEEE Access, vol. 7, pp. 57332-57340, May 2019.

[23] M. Alageli, A. Ikhlef, F. Alsifiany, M. A. M. Abdullah, G. Chen and J. Chambers, "Optimal Downlink Transmission for cell-free SWIPT
Massive MIMO Systems With Active Eavesdropping," IEEE Trans. Inf. Forensics Secur., vol. 15, no. 1, pp. 1983-1998, Jan. 2020.

[24] J. Zhu, D. W. K. Ng, N. Wang, R. Schober, and V. K. Bhargava, "Analysis and Design of Secure Massive MIMO Systems in the Presence of Hardware Impairments," IEEE Trans. Wireless Commun., vol. 16, no. 3, pp. 2001-2016, Mar. 2017.

[25] J. Zhu, Y. Li, N. Wang, and W. Xu, "Wireless Information and Power Transfer in Secure Massive MIMO Downlink With Phase Noise," IEEE Wireless Commun. Lett., vol. 6, no. 3, pp. 298-301, Jun. 2017.

[26] X. Zhang, D. Guo, K. An, and B. Zhang, "Secure Communications Over cell-free Massive MIMO Networks With Hardware Impairments," IEEE Syst. J., vol. 14, no. 2, pp. 1909-1920, Jun. 2020.

[27] Q. Xu, and P. Ren, "Secure Massive MIMO Downlink With LowResolution ADCs/DACs in the Presence of Active Eavesdropping," IEEE Access, vol. 8, pp. 140981-140997, Aug. 2020.

[28] E. Björnson, J. Hoydis, M. Kountouris, and M. Debbah, "Massive MIMO Systems With Non-Ideal Hardware: Energy Efficiency, Estimation, and Capacity Limits," IEEE Trans. Inf. Theory, vol. 60, no. 11, pp. 71127139, Nov. 2014

[29] L. Xu, X. Lu, S. Jin, F. Gao, and Y. Zhu, "On the Uplink Achievable Rate of Massive MIMO System with Low-Resolution ADC and RF Impairments," IEEE Commun. Lett., vol. 23, no. 3, pp. 502-505, Mar. 2019.

[30] Y. Zhang, M. Zhou, Y. Cheng, L. Yang, and H. Zhu, "RF Impairments and Low-Resolution ADCs for Nonideal Uplink cell-free Massive MIMO Systems," IEEE Syst. J., [Online] Doi:10.1109/JSYST.2020.2990709

[31] X. Hu, C. Zhong, X. Chen, W. Xu, H. Lin, and Z. Zhang, "cell-free Massive MIMO Systems With Low Resolution ADCs," IEEE Trans. Commun., vol. 67, no. 10, pp. 6844-6857, Oct. 2019.

[32] J. Zhang, Y. Wei, E. Björnson, Y. Han, and S. Jin, "Performance Analysis and Power Control of cell-free Massive MIMO Systems With Hardware Impairments," IEEE Access, vol. 6, pp. 55302-55314, Oct. 2018.

[33] A. K. Fletcher, S. Rangan, V. K. Goyal, and K. Ramchandran, "Robust Predictive Quantization: Analysis and Design Via Convex Optimization," IEEE J. Sel. Topics Signal Process., vol. 1, no. 4, pp. 618-632, Dec. 2007.

[34] O. T. Demir, and E. Björnson, "The Bussgang Decomposition of Nonlinear Systems: Basic Theory and MIMO Extensions [Lecture Notes]," IEEE Signal Process Mag., vol. 38, no. 1, pp. 131-136, Jan. 2021.

[35] Y. Zhang, M. Zhou, X. Qiao, H. Cao and L. Yang, "On the Performance of Cell-Free Massive MIMO With Low-Resolution ADCs," IEEE Access, vol. 7, pp. 117968-117977, Sept. 2019.

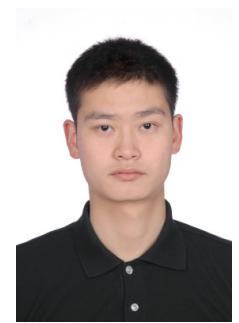

Xianyu Zhang received the B.E. degree and M.E. degree in Chongqing Communication Institute, Chongqing, China, in 2007 and 2010, the Ph D. degree in communication engineering from Army Engineering University of PLA, Nanjing, China, in 2019. His research interests focus on massive MIMO communication, physical layer security, cooperative relay networks, channel estimation, multiuser communication systems and green communication.

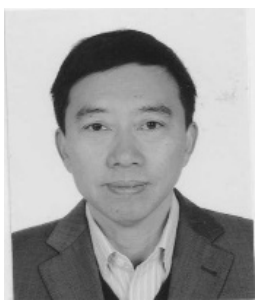

Tao Liang received the Ph.D. degree in computer science and technology from the Nanjing Institute of Communications Engineering, Nanjing, China, in 1998. He is currently a Research Fellow with the 63rd Research Institute, National University of Defense Technology, and also with the Nanjing Telecommunication Technology Institute, Nanjing. His research interests include satellite communication, digital signal processing in communications, physical layer security, cooperative communication, and cognitive networks. 


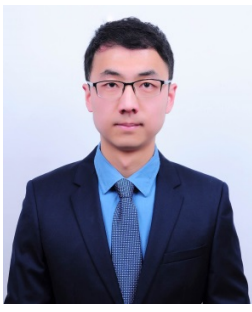

Kang An received the B.E. degree in electronic engineering from Nanjing University of Aeronautics and Astronautics, Nanjing, China, in 2011, the M.E. degree in communication engineering from PLA University of Science and Technology, Nanjing, China, in 2014, and $\mathrm{Ph} \mathrm{D}$. degree in communication engineering from Army Engineering University of PLA, Nanjing, China, in 2017. Since Jan. 2018, he is with National University of Defense Technology, Nanjing, China, where he is currently an Engineer. His current research interests are satellite communication, 5G mobile communication networks, cognitive radio and etc.

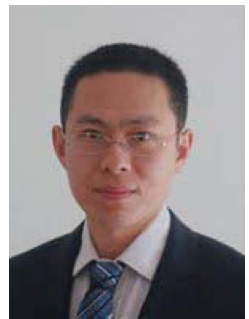

Gan Zheng (S'05-M'09-SM'12-F'21) received the BEng and the MEng from Tianjin University, Tianjin, China, in 2002 and 2004, respectively, both in Electronic and Information Engineering, and the $\mathrm{PhD}$ degree in Electrical and Electronic Engineering from The University of Hong Kong in 2008. He is currently Reader of Signal Processing for Wireless Communications in the Wolfson School of Mechanical, Electrical and Manufacturing Engineering, Loughborough University, UK. His research interests include machine learning for communications, UAV communications, mobile edge caching, full-duplex radio, and wireless power transfer. He is the first recipient for the 2013 IEEE Signal Processing Letters Best Paper Award, and he also received 2015 GLOBECOM Best Paper Award, and 2018 IEEE Technical Committee on Green Communications \& Computing Best Paper Award. He was listed as a Highly Cited Researcher by Thomson Reuters/Clarivate Analytics in 2019. He currently serves as an Associate Editor for IEEE Communications Letters and IEEE Wireless Communications Letters.

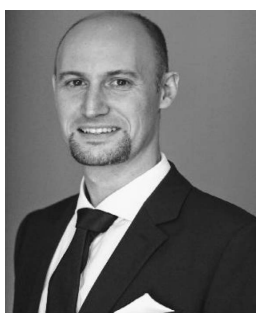

Symeon Chatzinotas (S06-M09-SM13) is currently Full Professor/Chief Scientist I and Head of the SIGCOM Research Group at SnT, University of Luxembourg. He is coordinating the research activities on communications and networking, acting as a PI for more than 20 projects and main representative for 3GPP, ETSI, DVB. In the past, he has been a Visiting Professor at the University of Parma, Italy, lecturing on "5G Wireless Networks". He was involved in numerous R\&D projects for NCSR Demokritos, CERTH Hellas and CCSR, University of Surrey. He was the co-recipient of the 2014 IEEE Distinguished Contributions to Satellite Communications Award and Best Paper Awards at EURASIP JWCN, CROWNCOM, ICSSC. He has (co-)authored more than 450 technical papers in refereed international journals, conferences and scientific books. He is currently in the editorial board of the IEEE Transactions on Communications, IEEE Open Journal of Vehicular Technology and the International Journal of Satellite Communications and Networking. 